\title{
Deutschland leidet unter einem Brain Drain
}

In Deutschland vollzieht sich quantitativ und qualitativ ein Wandel in der Struktur der Wanderungsbewegungen. Nach den Zahlen des Statistischen Bundesamtes standen im Jahr 2008682000 Zuzügen 738000 Fortzüge gegenüber. Damit sind in Deutschland zum ersten Mal seit der deutschen Vereinigung mehr Menschen aus- als eingewandert. Dies wäre nicht weiter beunruhigend, wenn diese Entwicklung auf die Finanzkrise zurückzuführen wäre. Dies ist jedoch nicht der Fall. In Deutschland ist bereits seit Mitte der 1990er Jahre ein kontinuierlicher Rückgang des Saldos aus $\mathrm{Zu}$ - und Fortzügen zu beobachten. So ist der Wanderungssaldo von durchschnittlich 318000 Personen p.a. in den 1990er Jahren seit dem Jahr 2000 auf durchschnittlich 129000 Personen p.a. gefallen. Auch die wirtschaftliche Erholung ab dem Jahr 2005 hat nicht zu einem Anstieg der Migration geführt.

Würde diese Entwicklung anhalten, ergäben sich gravierende Folgen für die demographische Struktur der Bevölkerung und die sozialen Sicherungssysteme. Nach den demographischen Projektionen des IAB würde unter optimistischen Annahmen über eine steigende Erwerbspartizipation von Frauen das Erwerbspersonenpotenzial in Deutschland ohne eine Nettozuwanderung von 44,5 Mio. Personen im Jahr 2005 auf 28 Mio. Personen im Jahr 2050 sinken. Bei einer Nettozuwanderung von 200000 Personen im Jahr - das entspräche dem langfristigen historischen Durchschnitt in Deutschland - könnte das Erwerbspersonenpotenzial im Jahr 2050 immerhin noch bei etwa 35 Mio. Personen stabilisiert werden. Eine Fortsetzung des gegenwärtigen Wanderungstrends würde dagegen erhebliche Folgen für die Finanzierung der sozialen Sicherungssysteme, insbesondere der Rentenversicherungen, aufwerfen.

Neben dem quantitativen Rückgang der Zuwanderung zeichnet sich ein schleichender, aber nicht minder beunruhigender Trend in der Qualifikationsstruktur der Wanderungsbewegungen ab. Immer mehr deutsche Staatsbürger mit mittleren und hohen Qualifikationen verlassen Deutschland. Dieser schon länger zu beobachtende Trend wurde statistisch lange durch die Zuwanderung von Spätaussiedlern nach Deutschland verdeckt. Die deutschen Auswanderer sind deutlich höher qualifiziert als der Durchschnitt der deutschen Bevölkerung. Zwar handelt es sich nicht nur um hoch qualifizierte Hochschulabsolventen, sondern in der Mehrheit um qualifizierte Facharbeiter. Dennoch ist der Anteil der Hochschulabsolventen deutlich höher als im Durchschnitt der Bevölkerung. Dies ist nicht weiter ungewöhnlich, weil die Wanderungsneigung in den meisten Ländern mit zunehmender Qualifikation steigt.

Der negative Wanderungssaldo der deutschen Bevölkerung reflektiert die zunehmende Internationalisierung der Arbeitsmärkte für qualifizierte und hoch qualifizierte Arbeitskräfte. Deutschland könnte von diesem Trend profitieren, wenn in gleichem Maße auch qualifizierte und hoch qualifizierte Arbeitskräfte nach Deutschland wandern würden. Dies ist jedoch nicht der Fall. Nach den vorliegenden Daten ist Deutschland ein Nettoexporteur von hoch qualifizierten Arbeitskräften. Im Zensusjahr 2000/01 lebten rund 940000 Deutsche mit Hochschulabschluss in anderen OECD-Ländern, denen 570000 Ausländer mit Hochschulabschluss aus der OECD in Deutschland gegenüberstanden. Damals konnte diese Differenz gerade noch durch die Zahl der in Deutschland lebenden Ausländer aus Nicht-OECD Staaten mit Hochschulabschluss ausgeglichen werden.

Nun hat Deutschland in den vergangenen Jahren durch verschiedene Reformen des Zuwanderungsrechts versucht, hoch qualifizierte Arbeitskräfte auch aus Drittstaa-

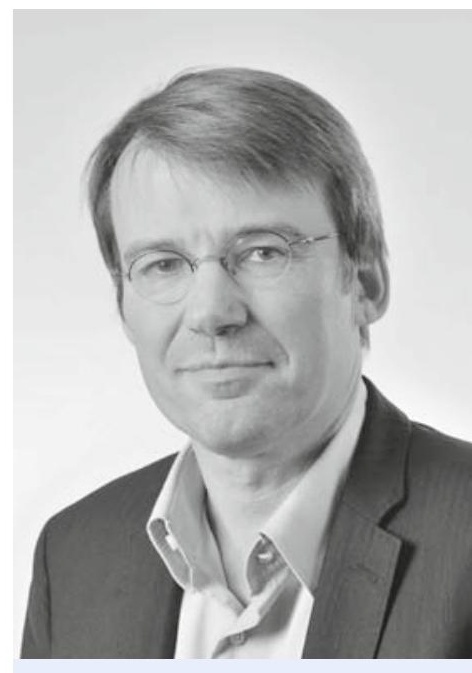

Herbert Brücker ist

Professor für Volkswirtschaftslehre an der Universität Bamberg und Leiter des Forschungsbereichs „Internationale Vergleiche und europäische Integration" im Institut für Arbeitsmarkt- und Berufsforschung (IAB). 
ten, d.h. aus Staaten, die nicht der Europäischen Union und dem Europäischen Wirtschaftsraum angehören, zu gewinnen. So wurde mit dem 2005 in Kraft getretenen Zuwanderungsrecht einer kleinen Gruppe von Hochqualifizierten wie Wissenschaftlern und Spitzensportlern ein Niederlassungsrecht eingeräumt. Darüber hinaus konnten Manager und hoch qualifizierte Spezialisten eine Aufenthalts- und Arbeitserlaubnis erhalten, sofern ihr Bruttoeinkommen das Doppelte der Beitragsbemessungsgrenze zur gesetzlichen Krankenversicherung (rund 85000 Euro) übersteigt. Schließlich besteht die Möglichkeit einer befristeten Arbeitserlaubnis für qualifizierte Arbeitskräfte, sofern der Nachweis geführt werden kann, dass diese Arbeitsplätze nicht mit Deutschen, EU-Ausländern und anderen bevorrechtigten Ausländergruppen besetzt werden können. Dies wird im Rahmen eines Zustimmungsverfahrens durch die Bundesagentur für Arbeit geprüft. Im Rahmen einer 2009 in Kraft getretenen Novellierung dieses Gesetzes wurde u.a. die Einkommensgrenze für Spezialisten und Manager auf die Beitragsbemessungsgrenze zur gesetzlichen Rentenversicherung gesenkt (rund 66000 Euro).

Diese vom früheren Bundesarbeitsminister Olaf Scholz als modernstes Zuwanderungsrecht Europas gelobten Regelungen haben sich jedoch als wirkungslos erwiesen. Bei 394596 Zuzügen im Jahr 2008 wurden insgesamt 1096 Aufenthaltserlaubnisse für neu eingereiste Hochqualifizierte, Wissenschaftler und Selbständige erteilt. Hinzu kommen weitere 24598 Aufenthaltserlaubnisse für neu eingereiste qualifizierte Arbeitskräfte, die eine Aufenthaltserlaubnis nach dem Zustimmungsverfahren der BA erhalten haben. Im Jahr 2009 ist die Zahl der Aufenthaltserlaubnisse für neu eingereiste Hochqualifizierte und Selbständige auf 1078 Personen und die Zahl der Aufenthaltserlaubnisse für neu eingereiste qualifizierte Arbeitskräfte auf 21010 Personen gesunken.

Angesichts dieser Entwicklungen bedarf es einer Neuorientierung der Einwanderungspolitik. Der globale Wettbewerb um hoch qualifizierte Arbeitskräfte steht zwar erst am Anfang, aber er wird sich in den kommenden Jahren deutlich verschärfen. Um diese zu gewinnen, müsste Deutschland die Eingangsbarrieren für Hochschulabsolventen, aber auch qualifizierte Facharbeiter deutlich senken. Die von der EU im Rahmen der Bluecard-Direktive vorgeschlagene Einkommensgrenze des 1,5-fachen des Medianeinkommens wäre eine sinnvolle Einkommensgrenze, weil sie in etwa den Anfangsgehältern von Hochschulabsolventen (rund 35000 Euro) entspricht.

Qualifizierte Zuwanderung bedeutet allerdings auch mehr Zuwanderung. Erhebliche Teile der Zuwanderung - wie die Wanderung innerhalb des Gemeinsamen Binnenmarktes der EU oder der Familiennachzug - lassen sich nicht bzw. nur begrenzt steuern. Bei einer jährlichen Zuwanderung von 400000 bis 600000 Personen müsste die Bruttozuwanderung um mindestens 200000 Personen pro Jahr ausgeweitet werden, wenn eine Veränderung der Qualifikationsstruktur der ausländischen Bevölkerung sichtbar werden soll. Eine solche Ausweitung kann nicht allein durch institutionelle Regelungen erreicht werden. Sie hängt auch von vielen ökonomischen, sozialen und kulturellen Faktoren ab. Die Chancen für eine qualifizierte Zuwanderung sollten jedoch in Deutschland nicht unterschätzt werden. Zwar hat Deutschland durch die Sprache einen Wettbewerbsnachteil gegenüber den angelsächsischen Ländern. Aber durch die Nähe zu Ost- und Mitteleuropa, die niedrigen Hochschulgebühren und die Leistungsstärke der exportorientierten Industrien kann Deutschland nach dem Ende der Finanzkrise wieder zu einem der attraktivsten Zielländer für Migranten in Europa werden. Um diese Chance zu nutzen, müssten bereits jetzt erhebliche

Herbert Brücker

Institut für Arbeitsmarkt- und Berufsforschung (IAB) herbert.bruecker@iab.de
Reformen des Zuwanderungsrechts und anderer für die Migration relevanter Institutionen vorgenommen werden. Wird diese Chance versäumt, ergeben sich auf mittlere und lange Sicht erhebliche Nachteile Deutschlands im zunehmenden Wettbewerb um qualifizierte Arbeitskräfte. 